\title{
Band positioning in boron nitride and metal-free photocatalysts via photoelectron- and UV-Vis diffuse reflectance spectroscopy
}

Ravi B. Shankar, ${ }^{a, b}$ Anna Hankin, ${ }^{a}$ Gwilherm Kerherve, ${ }^{c}$ Camille Petit ${ }^{a, b^{*}}$

aDepartment of Chemical Engineering, Imperial College London, South Kensington Campus, Exhibition Road, London SW7 2AZ, United Kingdom

aBarrer Centre, Imperial College London, South Kensington Campus, Exhibition Road, London SW7 2AZ, United Kingdom

'Department of Materials, Imperial College London, South Kensington Campus, Prince's Consort Road, London SW7 2BP, United Kingdom

${ }^{*}$ Corresponding author:

E-mail: camille.petit@imperial.ac.uk; Phone: +44 (0)20 75943182 (C. Petit) 


\section{Abstract}

New photocatalysts, particularly porous ones such as porous boron nitride, have emerged that exhibit complex structures and for which, there is limited knowledge of the electronic structure. Gaining insight into their complete band structure on the absolute energy scale will help assessing their suitability for a given photocatalytic reaction. To address this, we rationalise key concepts of band positioning alignment for both porous and non-porous semiconductors on the absolute energy scale. The approach employs a range of techniques generally accessible to many research groups. It involves a combination of spectroscopic techniques, namely X-ray photoelectron spectroscopy to determine the work function and valence band offset, and UV-Vis diffuse reflectance spectroscopy to measure the band gap. We apply this to present the complete band structure of boron nitride, in both porous and non-porous forms. We validate our methodology by comparing the experimentally obtained band structure for graphitic carbon nitride and amorphous boron, both amorphous semiconductors with a known band structure. We show how this can help predict possible photocatalytic reactions and demonstrate this in the context of $\mathrm{CO}_{2}$ photoreduction. With porous materials, such as porous BN, garnering increasing interest for photocatalytic applications, shedding light on their band structures could pave the way towards a methodical tuning and optimization of the photochemistry of these materials.

Keywords: boron nitride, band structure, work function, photocatalysis, porous materials. 


\section{Introduction}

Solar fuel production via photocatalysis has gained traction as a renewable pathway in the transition from a fossil fuel dominated energy infrastructure to sustainable energy technologies. ${ }^{1,2}$ Designing robust and sustainable photocatalysts that can efficiently harness sunlight to drive a heterogeneous photocatalytic reaction remains an on-going challenge in the field of solar fuels synthesis. To this end, researchers have recently started investigating a range of metal-free porous materials as potentially viable photocatalysts. Examples of such materials include: carbon nitrides ${ }^{3-7}$, covalent organic frameworks ${ }^{8-10}$, linear conjugated polymers ${ }^{11-14}$ and composites thereof ${ }^{15,16}$.

The band structure of many of these metal-free porous materials, remains unknown. This prevents any attempt to rationalise the activity of the photocatalysts. Yet, it is key to improve the design of such materials. In a recent study, we focused on porous boron nitride $(B N)^{17}$, a material that is gaining attention in the photocatalysis community., 17-21 Porous BN exhibits key properties of an ideal photocatalyst, with the compelling features being its tuneable chemistry/photochemistry, which facilitate a tailored band structure a priori. ${ }^{22}$ However, prior to this work ${ }^{17}$, porous $\mathrm{BN}$ had never been studied for $\mathrm{CO}_{2}$ photoreduction. In our study, we delved deeper into the photochemistry of porous BN by employing X-ray photoelectron spectroscopy in conjunction with UV-vis diffuse reflectance spectroscopy to align the position the conduction (CB) and valence (VB) bands, as well as the Fermi level, on the absolute energy scale vs. vacuum. Using these results, we proved that: (i) porous $\mathrm{BN}$ is an n-type semiconductor and (ii) the $\mathrm{CB}$ and $\mathrm{VB}$ of porous $\mathrm{BN}$ straddle the redox potentials of $\mathrm{CO}_{2} / \mathrm{CO}$ and $\mathrm{H}^{+} / \mathrm{H}_{2}$ respectively, yielding a significant reduction overpotential as the thermodynamic driving force for $\mathrm{CO}_{2}$ photoreduction. Prior to this study, there had been no reports in the literature of the complete band structure of porous BN on the absolute energy scale vs. vacuum.

For n-type photocatalysts, the Mott-Schottky method appears to be the preferred and most widely used technique for obtaining the conduction band minimum, from which the valence 
band position can be calculated through knowledge of the band gap (see Supplementary Information for details). ${ }^{23-28}$ However, this method usually requires immersion of the semiconductor in an aqueous electrolyte, which constitutes a bottleneck for obtaining the band structure of materials that suffer from chemical instability in aqueous media, such as the porous boron nitride studied in this paper $^{29,30}$, as well as other porous materials. Furthermore, Hankin et al. have shown that the validity of the Mott-Schottky method is restricted to a few ideal semiconductors. ${ }^{31}$ The complex structures of most photocatalysts violate nearly all of the assumptions behind the derivation of the Mott-Schottky equation and so a considerable degree of interpretation of interfacial impedance data is required. This method is thus unreliable, and any extracted parameters require corroboration by other techniques. ${ }^{31}$, 32 Additionally, the Mott-Schottky method was developed to measure a semiconductor's Fermi level and so is not a direct measurement of either the conduction band edge (in $n$-type semiconductors) or the valence band edge (in $p$-type semiconductors). The assumption that the Fermi level is located very close to either band edge can not necessarily be made a priori and hence spectroscopic techniques are required to locate the band positions. The method described herein, however, solely relies on measuring the surface property of the material directly through a combination of spectroscopic techniques, rather than as a deposition on a conducting substrate in the presence of an electrolyte.

With this in mind, we rationalise herein key concepts of band alignment in a simple manner that can be applied to new semiconductors, particularly metal-free porous ones. The concepts presented here are often assumed in other studies and we aim to make them easily accessible to the community working on emerging photocatalysts. While doing so, we provide insights into the photophysics of porous $\mathrm{BN}$, an emerging photocatalyst, We validate the methodology using semiconductors with known band structures, namely: bulk graphitic carbon nitride $\left(\mathrm{g}-\mathrm{C}_{3} \mathrm{~N}_{4}\right)$ and amorphous elemental boron. ${ }^{33-40}$ One can apply this methodology to other metal-free porous materials. Maheu et al. conducted a similar study to differentiate between the band structures of rutile and anatase phases of $\mathrm{TiO}_{2}$ powders, and 
examine how the electronic structures of powdered materials vary from that of single crystal models. ${ }^{41}$ With porous materials garnering increasing interest for photocatalytic applications, quantitative information about their band structures could pave the way towards more methodical tuning and optimization of the photochemistry of these materials. For instance, knowledge of the band edge positions on the absolute energy scale vs. vacuum relative to the redox potentials can provide valuable insight into the required band structure tuning strategy a priori - i.e. does one simply need to reduce the band gap of the material to enhance light absorption, as in the case of transitioning from $h$-BN to porous $\mathrm{BN}$, or is shifting either the $C B$ or VB required to ensure a sufficient reduction or oxidation overpotential to drive a particular reaction? In doing so, one can tailor their photocatalyst development and optimization strategies accordingly, as opposed to conducting ad hoc tuning or following a trial and error method. We propose at the end of the end of the study a workflow one can follow to study new photocatalysts for a specific reaction.

\section{Methodology}

\section{Aligning the band positions on the absolute energy scale vs. vacuum}

We present here the alignment of the band position on an absolute energy scale in Figure 1. This pictorial representation of the complete band structure of a general semiconductor highlights the techniques employed to determine the electronic properties. The detailed process is described in the Supporting Information. The readers are also directed to comprehensive mini reviews in the literature, such as the works of Cahen and Kahn $(2003)^{42}$, Ishii et al. $(1999)^{43}$ and Kahn $(2016)^{44}$, that present the theoretical concepts of the methodology. For simplicity, the band structure is represented with flat bands near the surface, which necessitates no net charge accumulation at or near the surface. Indeed, this is rarely observed in inorganic semiconductors, such as porous boron nitride investigated in this study, due to the presence of trapped charges in surface gap states arising from dangling bonds, defects and vacancies. ${ }^{44-46}$ We address the potential biases from band bending and spatial charge distribution in the proceeding data analysis. We note here that 
since the charge carriers are bound and trapped in a potential well, the energies relative to absolute vacuum are negative. Hence, the work function and valence band offset take negative values relative to absolute vacuum based on their definitions in equations (1) and (2) in the Supporting Information. We note that we have attempted to generate Mott-Schottky plots from impedance data on porous BN with no success (see details in the Supporting Information).

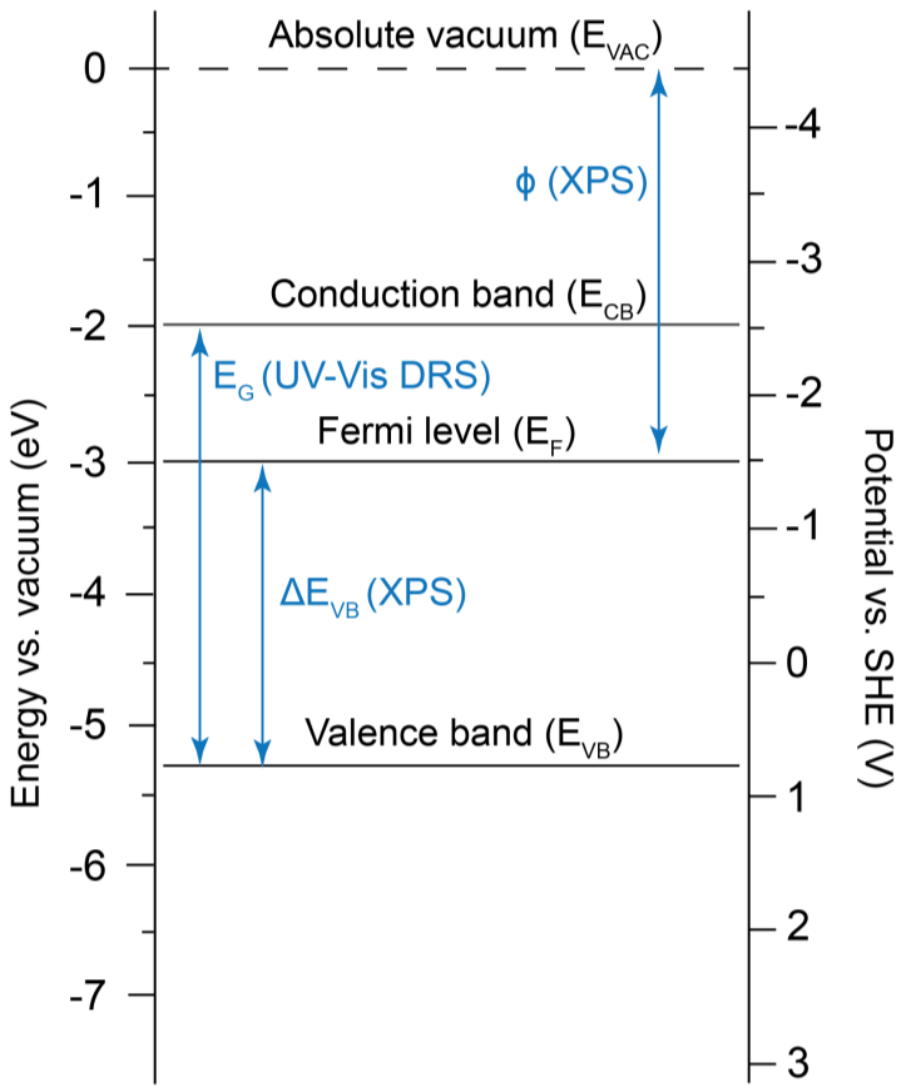

Figure $1 \mathrm{I}$ Schematic of the general semiconductor band structure, illustrating the outlined methodology. The work function $(\phi)$, optical band gap $\left(E_{G}\right)$, and valence band offset $\left(\Delta E_{V B}\right)$ are labelled with the corresponding techniques used to determine their values.

\section{Experimental Section}

\section{Materials}


Hexagonal boron nitride ( $h$-BN) and amorphous boron were obtained commercially: $h$-BN (Tres BN, Saint Gobain, max. particle size $74 \mathrm{~nm}$ ) and amorphous boron ( $\geq 95 \%$, SigmaAldrich).

Synthesis of porous BN. BN synthesis was based on the method developed by Marchesini et al.. ${ }^{47}$ In a typical synthesis, boric acid $\left(\mathrm{H}_{3} \mathrm{BO}_{3}\right.$, ACS reagent, $99.0 \%$, Sigma-Aldrich), urea $\left(\mathrm{CH}_{4} \mathrm{~N}_{2} \mathrm{O}\right.$, molecular biology grade, Sigma-Aldrich), and melamine $\left(\mathrm{C}_{3} \mathrm{H}_{6} \mathrm{~N}_{6}\right.$, ACS reagent, $99.0 \%$, Sigma-Aldrich), with a 1:1 molar ratio of boric acid to melamine and 1:5 molar ratio of boric acid to urea, were mechanically mixed together and finely ground for 5 minutes in an agate mortar to form a homogeneous powder. The finely ground powder was subsequently transferred to an alumina boat crucible, which was placed in a horizontal tubular furnace. The sample was initially degassed at ambient temperature for 3 hours under an inert nitrogen atmosphere (flow rate of $250 \mathrm{~cm}^{3} \mathrm{~min}^{-1}$ ). Once the degas was complete, the nitrogen flow rate was decreased to $50 \mathrm{~cm}^{3} \mathrm{~min}^{-1}$, and the sample was heated from ambient temperature to $1050^{\circ} \mathrm{C}$ with a ramp rate of $10{ }^{\circ} \mathrm{C} \mathrm{min}$. . This steady-state temperature was maintained for 3.5 hours, after which the samples were allowed to naturally cool to room temperature, whilst maintaining the same nitrogen flow rate. Upon completion of the synthesis, a porous white powder was obtained.

Synthesis of g- $C_{3} N_{4}$. In a typical synthesis, $12 \mathrm{~g}$ of melamine $\left(\mathrm{C}_{3} \mathrm{H}_{6} \mathrm{~N}_{6}\right.$, ACS reagent, $99.0 \%$, Sigma-Aldrich) were weighed and transferred to an alumina crucible, which was placed in a muffle furnace. The sample was heated from ambient temperature to $560{ }^{\circ} \mathrm{C}$ with a ramp rate of $5^{\circ} \mathrm{C} \mathrm{min}^{-1}$. This steady-state temperature was maintained for 4 hours, after which the sample was allowed to naturally cool to room temperature. Upon completion of the synthesis, a yellow solid product was obtained, which was subsequently ground in an agate mortar to form a fine, homogeneous powder. 


\section{Methods}

Fourier Transform infrared spectroscopy (FT-IR). The samples were first ground to a powder using an agate mortar. Subsequently, the spectra were obtained in the range of $500-4000$ $\mathrm{cm}^{-1}$ using a Perkin-Elmer Spectrum 100 FT-IR spectrometer equipped with an attenuated total reflectance (ATR) accessory.

Powder X-ray diffraction (XRD) was performed using a PANalytical X'Pert Pro X-ray diffractometer in reflection-transmission mode with a spinning stage (2 revolutions/second). An anode voltage of $40 \mathrm{kV}$ and emission current of $20 \mathrm{~mA}$ were chosen as the operating conditions using a monochromatic $\mathrm{Cu}-\mathrm{K} \alpha$ radiation source $(\lambda=1.54178 \AA)$. The X'Celerator silicon strip detector was used in the diffractometer.

Porosity measurements were performed using a porosity and surface area analyser (Micrometrics 3 Flex) via $\mathrm{N}_{2}$ sorption at $-196^{\circ} \mathrm{C}$. The samples were initially degassed overnight at $120^{\circ} \mathrm{C}$ at approximately 0.2 mbar pressure. Subsequently, prior to the sorption isotherm measurement, the samples were degassed in-situ for 4 hours at $120^{\circ} \mathrm{C}$. The equivalent specific surface areas of the samples were determined using the BrunauerEmmett-Teller (BET) method. ${ }^{48}$ The total pore volume was ascertained from the volume of $\mathrm{N}_{2}$ adsorbed at a relative pressure $\left(\mathrm{P} / \mathrm{P}_{0}\right)$ of 0.97 . The micropore volume was determined using the Dubinin-Radushkevich model. ${ }^{49}$

$X$-ray photoelectron spectroscopy (XPS) was employed to determine the relative elemental composition of the samples, the chemical states of the elements, the valence band off-set and secondary electron cut-off. This was conducted using a Thermo Scientific K-Alpha+ Xray Photoelectron Spectrometer equipped with a MXR3 AI K $\alpha$ monochromated X-ray source $(\mathrm{h} v=1486.6 \mathrm{eV})$. The samples were initially ground and mounted onto an XPS sample holder using a small rectangular piece of conductive carbon tape. The X-ray gun power was set to $72 \mathrm{~W}$ (6 mA and $12 \mathrm{kV}$ ). Survey scans were acquired using $200 \mathrm{eV}$ pass energy, 0.5 eV step size and $100 \mathrm{~ms}$ (50 ms $\times 2$ scans) dwell times. All of the high resolution core level spectra (B 1s, N 1s, C 1s, and O 1s) were obtained using a $20 \mathrm{eV}$ pass energy and $0.1 \mathrm{eV}$ step size. The valence band spectra were obtained using a $15 \mathrm{eV}$ pass energy and $0.05 \mathrm{eV}$ 
step size. The results were analysed using the Thermo Avantage data analysis program. Any charging effect in the core level and valence band measurements was mitigated by using a dual-beam flood gun that uses the combination of low energy electrons and argon ions.

UV-Vis diffuse reflectance spectroscopy (UV-Vis DRS) was employed to determine the optical band gap of the photocatalysts investigated in this study. The DR-UV/Vis spectra were measured using a Agilent Cary 500 UV-vis-NIR spectrometer equipped with an integrating sphere. Spectral band width was set to $2 \mathrm{~nm}$, with Spectralon as a standard. The samples were compressed onto a $\mathrm{KBr}$ backed pellet for analysis. Spectra were treated using Kubelka-Munk function ${ }^{50}$ in order to eliminate any tailing contribution from the DR-UV-vis spectra. The following equation was applied: $F(R)=(1-R)^{2} / 2 R$, where $R$ is the reflectance. The band-gap $\left(E_{g}\right)$ values were estimated from the Tauc plot of $F(R) \cdot h v^{1 / 2}$ versus photon energy by extrapolating the linear region.

Work function measurements (XPS). The work function of porous BN was determined by measuring the secondary electron cut-off in the low kinetic energy region using a Thermo Scientific K-Alpha ${ }^{+}$X-ray Photoelectron Spectrometer equipped with a MXR3 Al K $\alpha$ monochromated X-ray source $(\mathrm{h} v=1486.6 \mathrm{eV})$. A known mass $(100 \mathrm{mg})$ of sample was pelletized to form a thin, homogeneous pellet, which was clipped to the sample holder. The sample holder contained a clean gold standard sample, which was used as a reference material to ensure correct calibration. A bias of $-30 \mathrm{~V}$ was applied to the samples using a DC supply and the cut-off spectra were obtained using a pass energy of $10 \mathrm{eV}$. The connection is made at the bottom of the sample holder and the voltage is activated from the sputter window. To account for potential variations across the surface of the material, the work function was measured at four different locations and an average was taken. The data for the secondary electron cut-off has been processed such that the tangent is taken at the segment of the graph where the data exhibits a linear trend after the initial curve upwards from the x-axis. To do so, we have used linear extrapolation to fit a line of best fit to the linear data range with the corresponding equation and coefficient of determination. 


\section{Results}

\section{Band structures of the materials}

Prior to determining the band structures of the studied materials, we characterised their chemical and physical features using Fourier transform infrared spectroscopy (FT-IR), X-ray photoelectron spectroscopy (XPS), X-ray diffraction (XRD) and nitrogen sorption at $-196{ }^{\circ} \mathrm{C}$. The results of these analyses are presented in Figures S1-S7 and Tables S1-S2. All techniques confirmed the expected nature of the materials.

We then probed the optoelectronic properties of the studied materials through UV-vis diffuse reflectance spectroscopy. From the Tauc plot of the transformed Kubelka-Munk function against photon energy (Figure 2a), porous BN exhibits a UV-range optical band gap of 4.20 $\mathrm{eV}$, significantly lower than that of $h-\mathrm{BN}(5.50 \mathrm{eV})$. Bulk $\mathrm{g}-\mathrm{C}_{3} \mathrm{~N}_{4}$ displays a visible-range optical band gap of $2.74 \mathrm{eV}$. Amorphous boron has the lowest band gap of $1.60 \mathrm{eV}$, which extends its light absorption capability through the full UV-visible spectrum and extends into the IR region. The values of the optical band gaps for our reference materials are consistent with reports in the literature..$^{7,17,28,39,40,51-54}$

We next conducted valence band XPS measurements to determine the valence band offset $\left(\Delta E_{V B}\right)$ for each of the samples (Figure $\left.2 \mathrm{~b}-2 \mathrm{e}\right)$. High resolution core level spectra for porous $\mathrm{BN}$ and $h$-BN (B 1s, N 1s, C 1s, and $\mathrm{O} 1 s$ ), bulk $\mathrm{g}-\mathrm{C}_{3} \mathrm{~N}_{4}$ (C $1 s$ and $\mathrm{N} 1 s$ ) and amorphous boron (B 1s, C 1s, O 1s) were simultaneously measured along with the valence band spectrum. No effects of charging were observed (core level spectra are shown in Supplementary Figures 1-4). In the event of charging, the peak centres of the core level photoemission spectra would shift towards higher binding energies due to an accumulation of positive charge. ${ }^{55,56}$ The valence band offsets for $\mathrm{g}^{-} \mathrm{C}_{3} \mathrm{~N}_{4}$ and amorphous boron were 
determined to be $-2.21 \mathrm{eV}$ and $-0.25 \mathrm{eV}$ respectively, which aligns well the literature reports. ${ }^{51,57,58}$
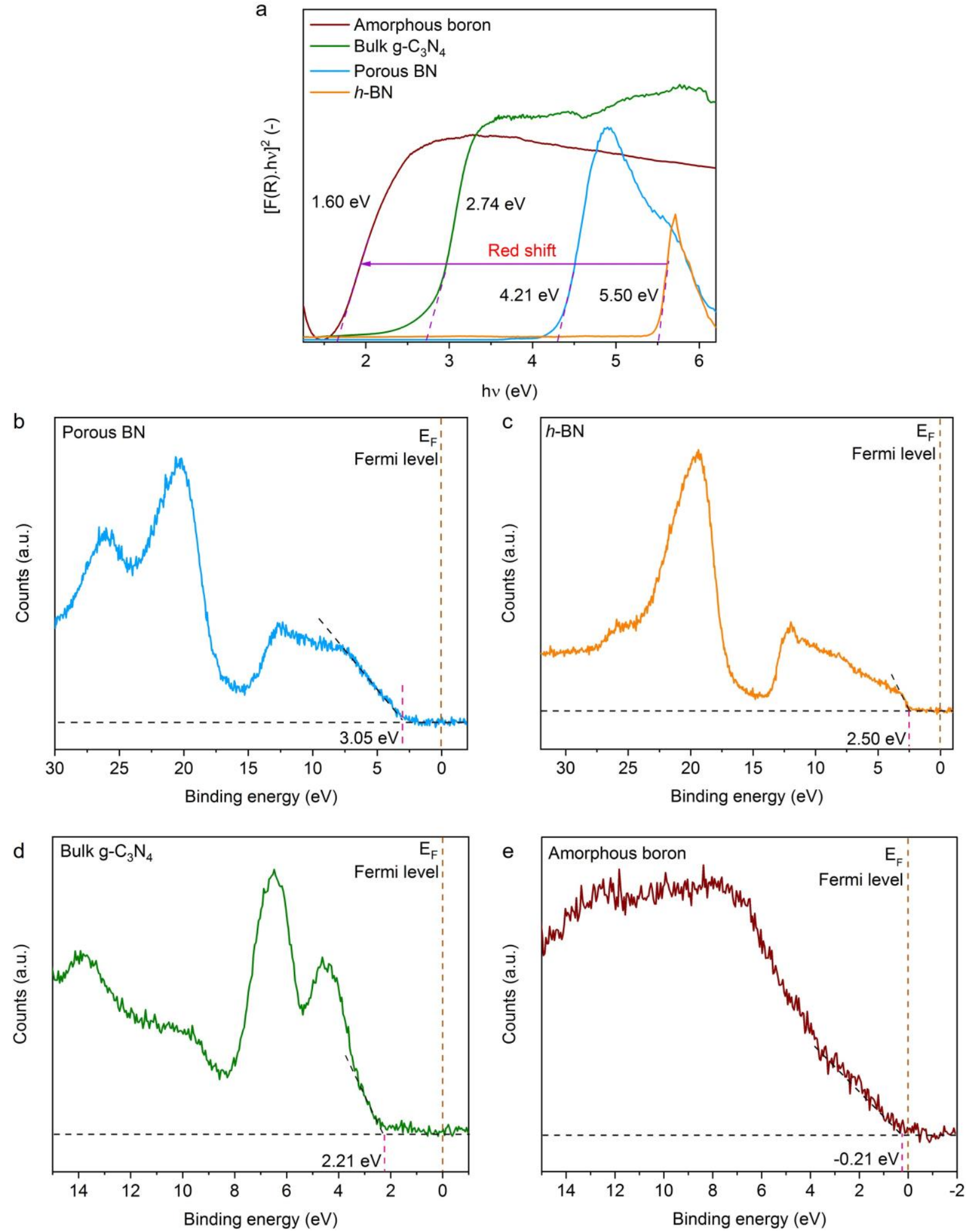

Figure 2 I Optical band gap and valence band offset measurements for porous BN, $\boldsymbol{h}$ $\mathrm{BN}, \mathbf{g}-\mathrm{C}_{3} \mathrm{~N}_{4}$, and amorphous boron determined through UV-Vis diffuse reflectance 
spectroscopy. (a) Tauc plot of the Kubelka-Munk function versus photon energy to determine the optical band gap of the materials, with the red shift highlighted. Valence band offset of (b) porous $\mathrm{BN},(\mathbf{c}) h-\mathrm{BN}$, (d) bulk g- $\mathrm{C}_{3} \mathrm{~N}_{4}$ and (e) amorphous boron. Note that the negative sign for $\Delta \mathrm{E}_{\mathrm{VB}}$ has been omitted here as binding energies above the Fermi level (positioned at a binding energy of $0 \mathrm{eV}$ ) are positive.

We note that work function measurements conducted through photoelectron spectroscopy are extremely sensitive to subtle changes in experimental conditions ${ }^{59-61}$ (e.g. pre-analysis surface treatments involving heat or chemicals, sputtering with an ion gun, presence of surface impurities etc.) and necessitate the preparation of a well-controlled, homogeneous surface. ${ }^{44,59,62}$ To negate the effects of surface roughness, we pelletized a known mass of each sample and formed a thin homogeneous surface. Further, to account for potential variations across the surface of the material, we measured the work function at four different locations and took an average. As previously discussed, the work function consists of both a bulk and surface dipole component, with the latter related to the redistribution of charges on the surface of the material and the solid-vacuum interface. ${ }^{44}$ This redistribution of charges is sensitive to the atomic arrangement on the surface of the solid. In this regard, net charge accumulation stemming from the presence of trapped charges in surface gap states, arising from the high concentration of defects and vacancies, may play an important role in the surface energetics of porous materials and can lead to band bending. ${ }^{42,43,63}$

The average work functions for porous $\mathrm{BN}, h-\mathrm{BN}$, bulk $\mathrm{g}-\mathrm{C}_{3} \mathrm{~N}_{4}$ and amorphous boron are shown in Figure 3a and Supplementary Table S3. Typical secondary electron cut-off spectra for the materials investigated are shown in Figures $3 b-3 e$. Bulk $g-C_{3} N_{4}$ exhibits the highest work function of $-4.8 \mathrm{eV}$ amongst the tested materials, this value is in good agreement with those reported in literature. ${ }^{51,64,65}$ This implies that of the materials studied, bulk $\mathrm{g}-\mathrm{C}_{3} \mathrm{~N}_{4}$ requires the largest input of energy to eject the bound electron from the core level. The lower work functions of the other materials may be associated with the formation of surface dipoles and/or a high concentration of surface defects. ${ }^{43-46,66}$ 

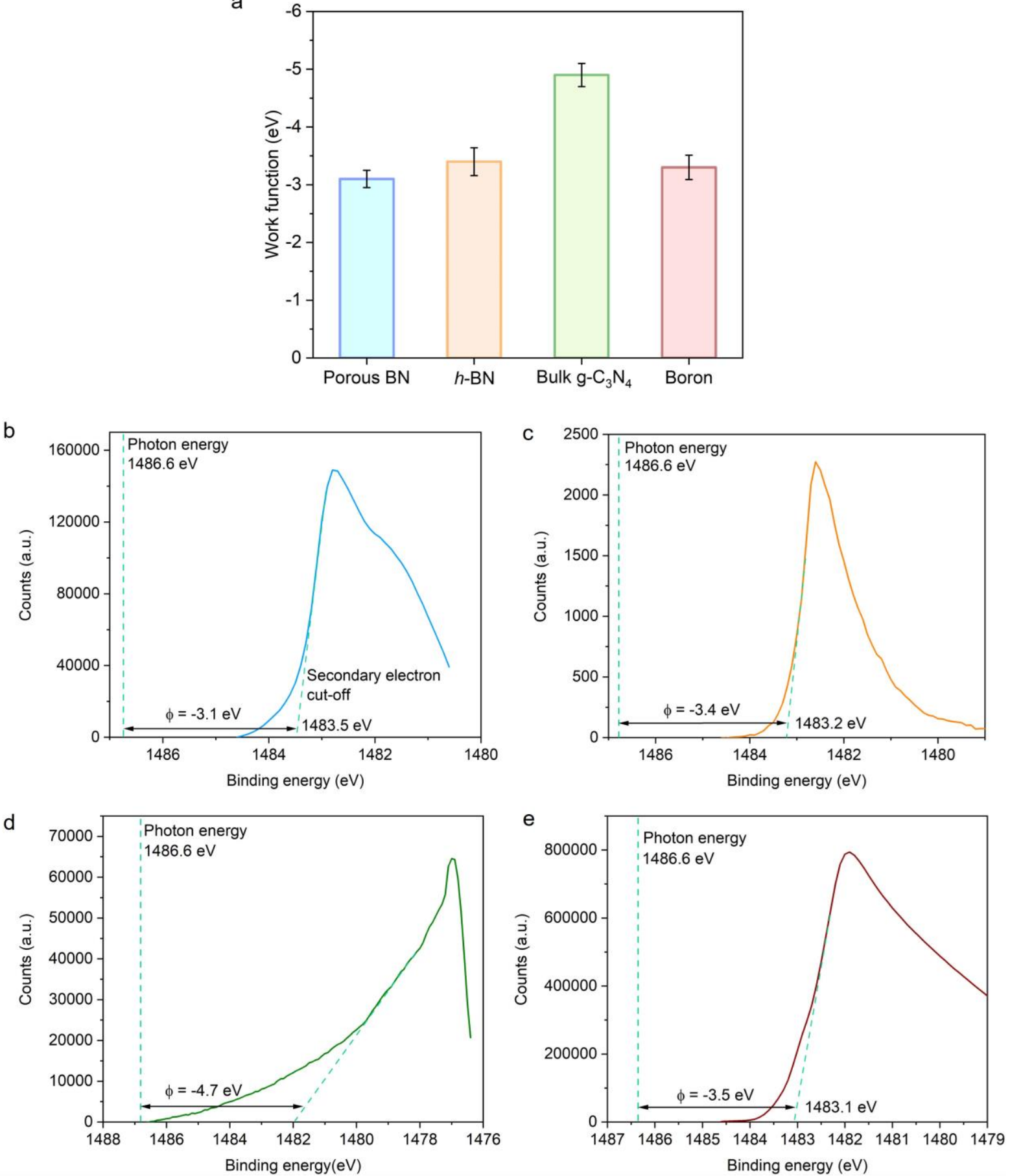

Figure 3 I Work function measurements and secondary electron cut-off spectra for porous $\mathrm{BN}, \boldsymbol{h}$-BN, bulk $\mathbf{g}-\mathrm{C}_{3} \mathrm{~N}_{4}$ and amorphous boron. (a) Average work functions of porous $\mathrm{BN}, h$-BN, bulk g- $\mathrm{C}_{3} \mathrm{~N}_{4}$ and amorphous boron taken over four opposite locations on the sample to account for potential variations across the surface. The work functions are labelled as negative here to stay consistent with the notations in equation (1). A typical secondary electron cut-off spectra for (b) porous $\mathrm{BN},(\mathbf{c}) h-\mathrm{BN}$, (d) bulk $\mathrm{g}-\mathrm{C}_{3} \mathrm{~N}_{4}$, and (e) amorphous boron. 
To align the absolute energy scale vs. vacuum with the redox potential scale vs. the standard hydrogen electrode (SHE), we used the fact that $-4.44 \mathrm{eV}$ on the former corresponds to $0.00 \mathrm{~V}$ on the latter at $298 \mathrm{~K}$ and $\mathrm{pH}=0 .{ }^{67}$ In doing so, we can align the known redox potentials for $\mathrm{CO}_{2} / \mathrm{CO}(-0.11 \mathrm{~V})$ and $\mathrm{H}^{+} / \mathrm{H}_{2}(0.00 \mathrm{~V})$ reported in the literature on the absolute energy scale. ${ }^{68}$ The redox potential for $\mathrm{CO}_{2} / \mathrm{CO}$ is that measured at $298 \mathrm{~K}$ in water as per the work of Bratsch. ${ }^{69}$ It is commonly used for the study of both aqueous and gaseous systems as the value for the gaseous system is not available. Combining this data with the values in Figures 2 and 3, we construct the complete band structure on the absolute energy scale vs. vacuum for the four materials (Figure 4).

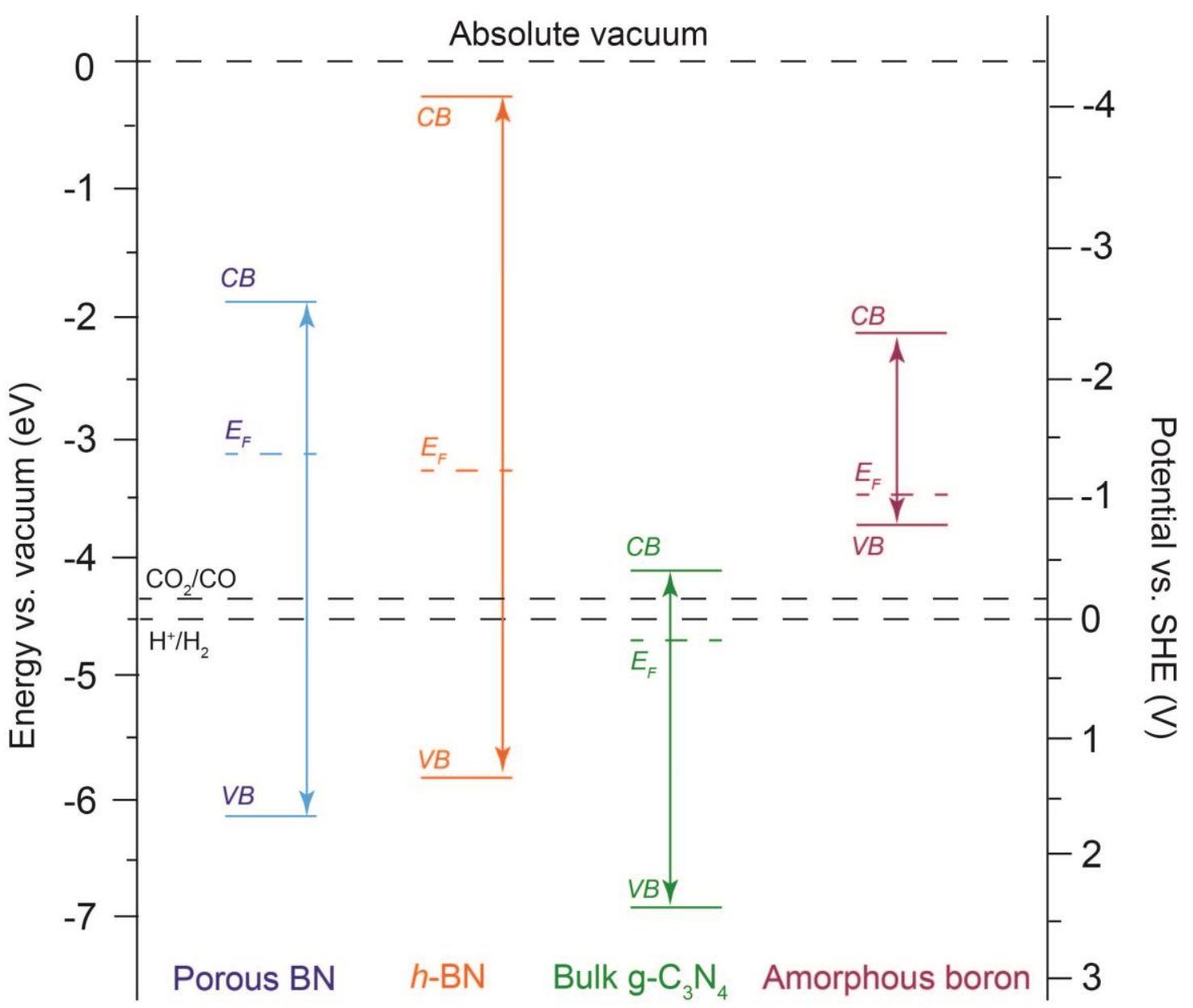

Figure 4 I Complete band structure of materials investigated in this study on the absolute energy scale with the conduction and valence bands, Fermi level and redox potentials for $\mathrm{CO}_{2} / \mathrm{CO}$ and $\mathrm{H}^{+} / \mathrm{H}_{2}$ at $\mathrm{pH}=0$ indicated. 
The band structure shows that the $\mathrm{CB}$ and VB of porous $\mathrm{BN}$ and $h$-BN straddle the redox potentials of $\mathrm{CO}_{2} / \mathrm{CO}$ and $\mathrm{H}^{+} / \mathrm{H}_{2}$ respectively, yielding a significant reduction overpotential as the thermodynamic driving force for $\mathrm{CO}_{2}$ photoreduction (Figure 4). Note that, we refer to the reduction overpotential as the difference in potential between the conduction band of the semiconductor and the redox potential of $\mathrm{CO}_{2} / \mathrm{CO}$. In addition, the Fermi level in porous BN was shown to lie closer to the conduction band than the valence band, which demonstrates that porous BN behaves as an n-type semiconductor.

\section{Validation of band structure of bulk $\mathrm{g}-\mathrm{C}_{3} \mathrm{~N}_{4}$ and amorphous boron with literature}

To validate the results obtained from this experimental procedure, we compare the obtained parameters in the band structures of bulk graphitic carbon nitride $\left(\mathrm{g}-\mathrm{C}_{3} \mathrm{~N}_{4}\right)$ and amorphous boron with the reported values in the literature (Figure 5). ${ }^{33-36}$ The positions of the conduction and valence bands, as well as the position of the Fermi level, in the experimentally obtained band structure for bulk $\mathrm{g}-\mathrm{C}_{3} \mathrm{~N}_{4}$ lie within the same range as the literature values but still exhibit some deviation. For example, Zhang and Antoinetti synthesised a bulk $\mathrm{g}-\mathrm{C}_{3} \mathrm{~N}_{4}$ material through the polycondensation of dicyanamide at $520^{\circ} \mathrm{C}$, and reported $\mathrm{CB}$ and VB positions at $-0.83 \mathrm{~V}$ and $1.83 \mathrm{~V}$, which deviate by approximately $0.4 \mathrm{~V}$ from the values reported using our methodology ( -0.45 $\mathrm{V}$ and $2.25 \mathrm{~V}$ from Figure 5). ${ }^{7}$ Similarly, Lin et al. synthesised a bulk $\mathrm{g}-\mathrm{C}_{3} \mathrm{~N}_{4}$ material from guanidine hydrochloride and reported the $\mathrm{CB}$ and $\mathrm{VB}$ to be at $-0.90 \mathrm{~V}$ and $1.78 \mathrm{~V}$ (Figure 5). $545454545454 \mathrm{We}$ attribute the differences to the different starting precursors and reaction temperatures, which can change the sample morphology and/or surface/bulk chemistry. 707070707070 To our best knowledge, the complete band structure of amorphous boron is yet to be reported in the literature. In fact, this material has only recently been applied as a photocatalyst. ${ }^{52}$ However, we compare here the experimental values we obtained for the individual components of the band structure, namely the band gap, valence band offset and work function to literature reports. The experimental optical band gap of $1.60 \mathrm{eV}$ corresponds well with literature values of $\sim 1.60 \mathrm{eV}$ 
of Liu et al. and $1.43 \mathrm{eV}$ of Shirai. ${ }^{39,40}$ Amorphous boron exhibits a very small valence band offset of $0.21 \mathrm{eV}$, which is in agreement with the literature value of $0.23 \mathrm{eV}$ measured by Zhou et al. ${ }^{38}$ King et al. investigated thin films of amorphous boron deposited on copper substrates and reported a similarly small valence band offset of 0.40 eV. ${ }^{585858585858}$ The small valence band offset indicates the strong p-type nature of amorphous boron with the Fermi level lying very close to the valence band maximum. The largest discrepancy was observed for the work function of amorphous boron, as shown in Figure 5. Madelung et al. and Knežević et al. reported work functions for pure amorphous boron thin films of $4.3 \mathrm{eV}$, whilst our experimental data indicates that a work function of $3.1 \mathrm{eV}$. ${ }^{37,71} 37,7137,7137,7137,7137$, ${ }^{71}$ We attribute this discrepancy to amorphous boron in our study not being etched. Diluted acid can indeed be used prior to XPS analysis to remove the amorphous oxide layer and other impurities and to obtain pure thin films of boron on metal substrates. The variation in sample preparation and the presence of impurities could give rise to contrasting work functions with XPS being a surface-sensitive analysis technique. ${ }^{44}, 62$ This gives us confidence that the methodology proposed in this study can be applied to a wide range of photocatalysts. Further, the analysis implies that bulk $\mathrm{g}-\mathrm{C}_{3} \mathrm{~N}_{4}$ exhibits the most n-type nature of the materials investigated in this study due to the smallest distance between the Fermi level and the conduction band $\left(-0.53 \mathrm{eV}\right.$ for $\mathrm{g}-\mathrm{C}_{3} \mathrm{~N}_{4}$ compared to $-1.16 \mathrm{eV}$ for porous $\left.\mathrm{BN}\right)$. The strong n-type behaviour supports for its application as a visible-light photocatalyst for $\mathrm{CO}_{2}$ photoreduction and $\mathrm{H}_{2}$ evolution. ${ }^{6,28,72-74}$ 


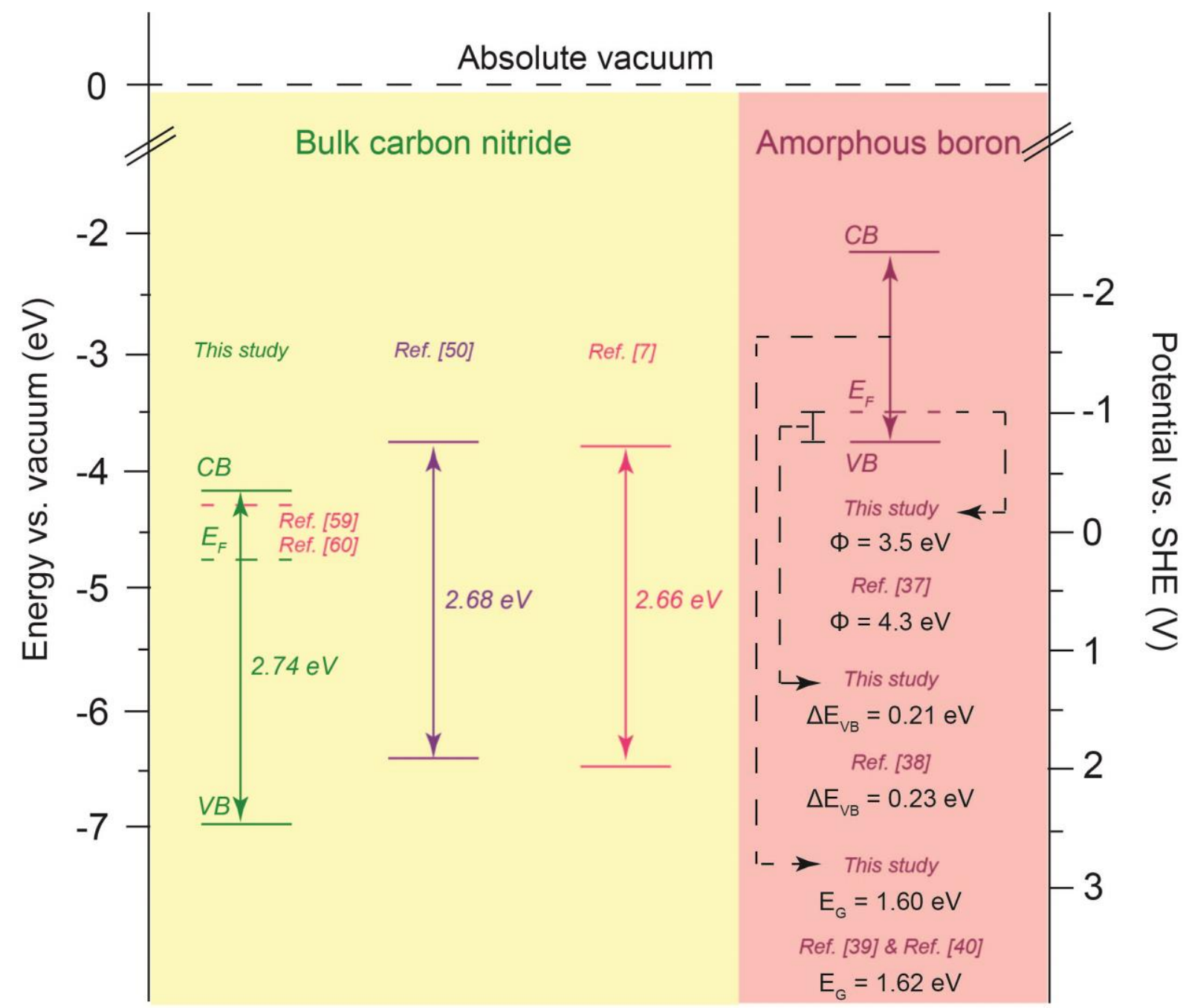

Figure 5 I Comparison of experimental band structure properties of bulk $g-C_{3} N_{4}$ and amorphous boron ${ }^{37-40}$ on the absolute energy scale with the literature values. ${ }^{7,37-40,54,64,65}$

\section{Parameter map for $\mathrm{CO}_{2}$ photoreduction performance vs. physical-chemical properties}

In our previous study, we investigated porous BN as a heterogeneous photocatalyst for combined $\mathrm{CO}_{2}$ capture and gas-phase photoreduction to $\mathrm{CO}$ and compared the specific $\mathrm{CO}$ production rate to reference materials $\left(\mathrm{P} 25 \mathrm{TiO}_{2}\right.$, bulk $\mathrm{g}-\mathrm{C}_{3} \mathrm{~N}_{4}$ and $\left.h-\mathrm{BN}\right) .{ }^{17}$ Having established the band structure of the materials investigated in this study, we proceeded to develop a parameter map relating the $\mathrm{CO}$ production rate reported for each material in our previous study against the reduction overpotential $\left(n_{\mathrm{co} / \mathrm{co}}\right)$ and the $\mathrm{CO}_{2}$ adsorption capacity (Figure 6). We chose the reduction overpotential and $\mathrm{CO}_{2}$ adsorption capacity as the 
independent variables in the parameter space as the former constitutes the thermodynamic driving force available to facilitate the $\mathrm{CO}_{2}$ photoreduction reaction, whilst the latter governs how much $\mathrm{CO}_{2}$ is able to be captured to be converted to $\mathrm{CO}$. To maximize the catalyst output for $\mathrm{CO}_{2}$ photoreduction, an ideal photocatalyst should desirably be positioned towards the shaded "desired region" in the parameter map (Figure 6). In doing so, the structure and morphology of the material enables good $\mathrm{CO}_{2}$ uptake, whilst its band structure provides a large thermodynamic driving force to drive the photoreduction of $\mathrm{CO}_{2}$.

We note that Figure 6 does not account for all parameters that may influence photocatalysis and particularly the band gap. Furthermore, the conditions of the band position measurements in Figure 4 do not reflect those of the actual $\mathrm{CO}_{2}$ photoreduction tests, i.e. non-vacuum atmosphere and illumination. Exposure to gaseous and adsorbed $\mathrm{CO}_{2}$, could influence the measured work function, and hence the conduction and valence band positions. ${ }^{43,}{ }^{44}$ Further, alongside reaction environment conditions, molecular orientation of the adsorbed molecules can have an impact on the XPS measurements due to charge displacement and the structure and density of surface dipoles. ${ }^{75},{ }^{76}$ In-situ XPS measurements could be employed to incorporate factors such as the presence of $\mathrm{CO}_{2}$ and light irradiation for more accurate measurements. This non-trivial approach relies on the availability of specialised equipment and reaction cells. The method outlined in this study remains a viable option for a 'quick' assessment, especially for relative band positioning. 


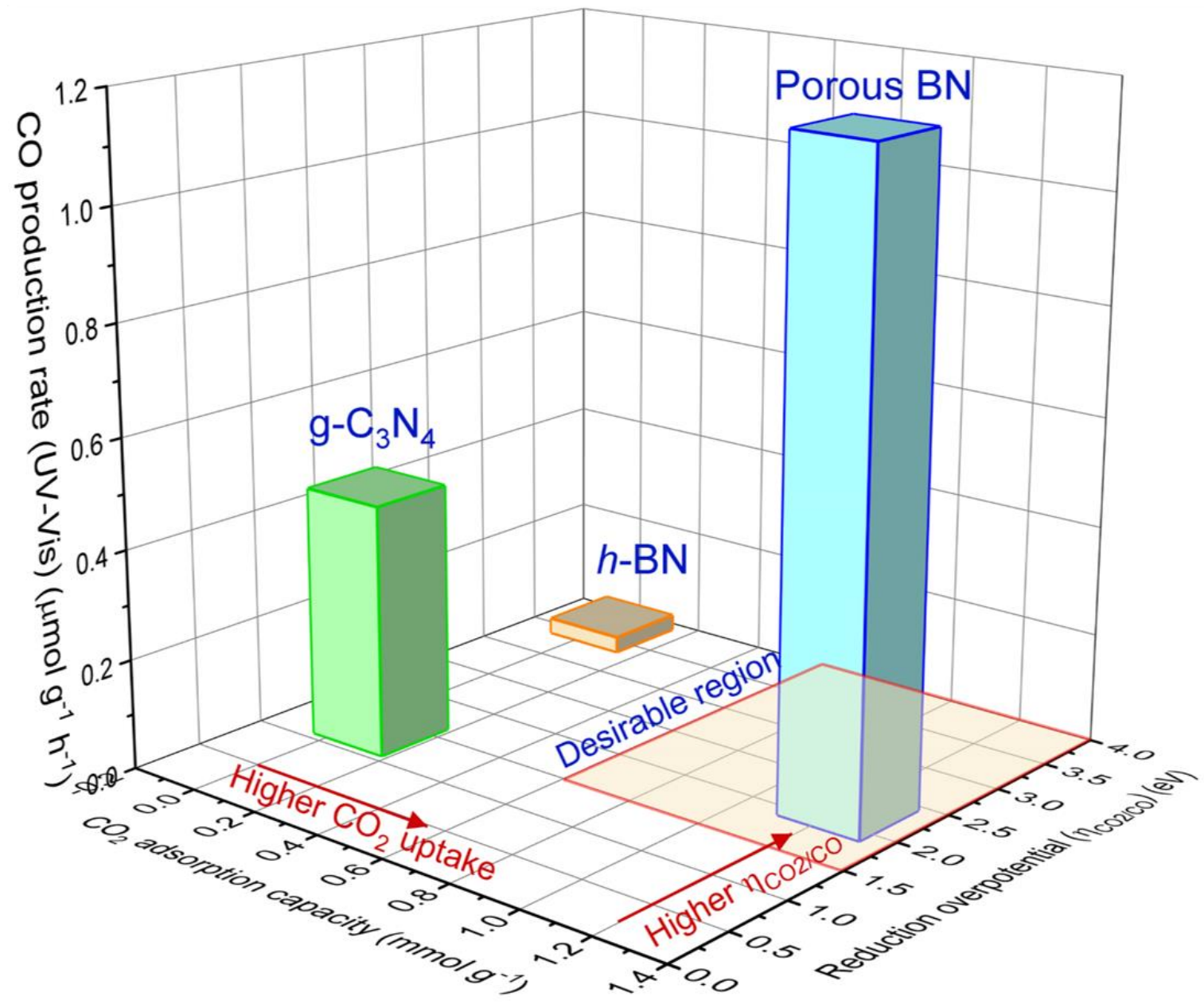

Figure 6 I Parameter map of the CO production rate as a functional of relevant materials properties, i.e.: reduction overpotential, obtained in this study, and $\mathrm{CO}_{2}$ adsorption capacity reported with the $\mathrm{CO}$ production rate in our previous study. ${ }^{17}$ The "desired region", where the photocatalyst offers both high $\mathrm{CO}_{2}$ capture and a large reduction overpotential to drive the subsequent photoreduction, is shaded and labelled.

\section{Application of methodology for photocatalyst screening}

To implement the methodology outlined in this study in the context of screening photocatalysts for the suitability towards a reaction, we envisage the workflow presented in Figure 7. We note that the work function can be obtained using different techniques such XPS, UPS or Kelvin probe measurements, and we chose the former in this particular study. If the material satisfies all of the criteria, the conduction and valence bands straddle the redox potential in the reaction system, which confirms that the reaction is thermodynamically 
favourable. By applying this workflow, one could identify the suitability of materials for given photocatalytic reactions simply through a set of three experiments. In the event that the material does not straddle the redox potentials, one could 'move' the position of the material into the "desired region" (Figure 6), by tailoring the band structure through for instance doping or forming composite materials (Figure 7).

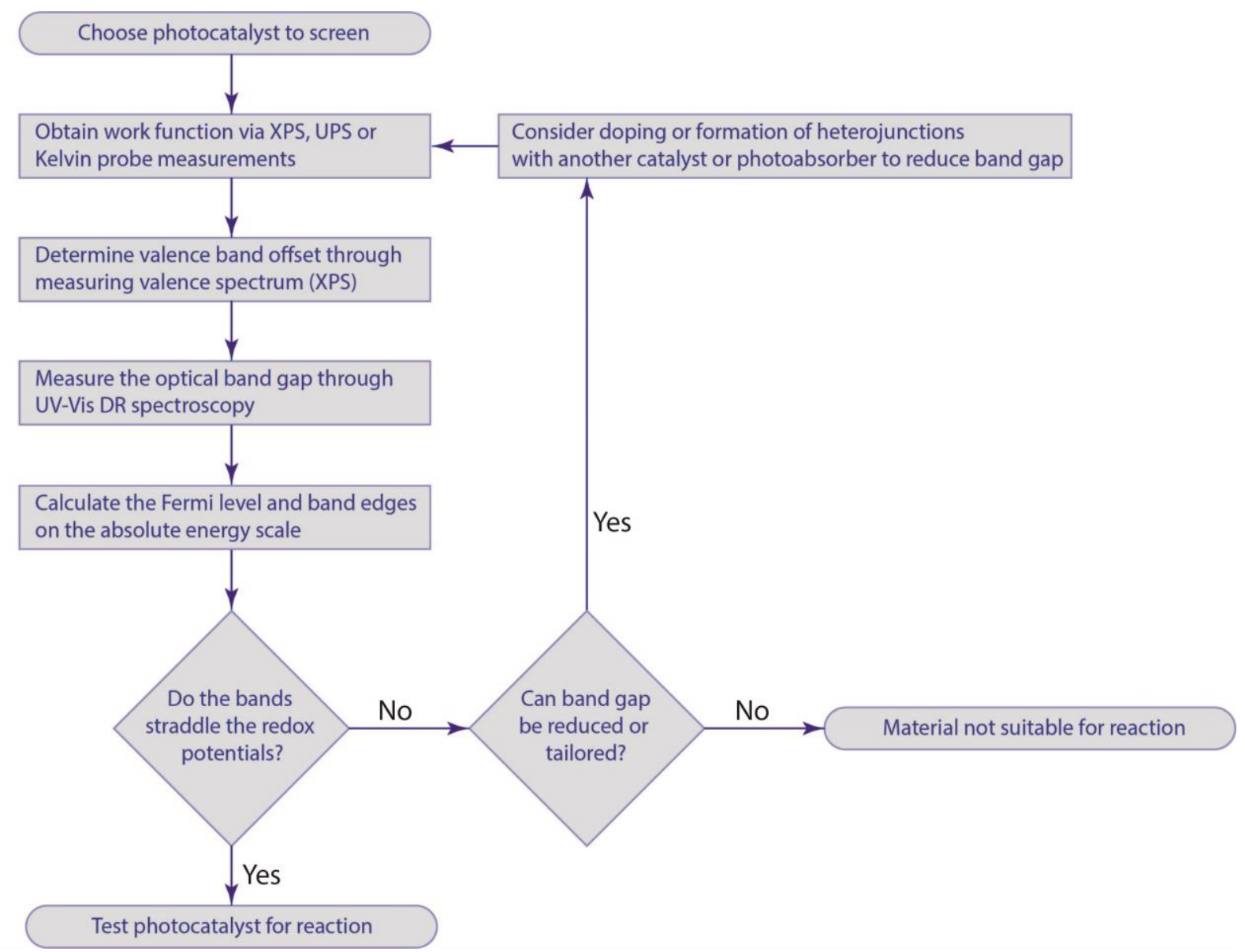

Figure 7 I Proposed workflow for application of experimental methodology for photocatalyst screening. If the material can satisfy all of the criteria, the conduction and valence bands straddle the redox potential in the reaction system, which confirms from a thermodynamic argument that it can be employed to facilitate the particular reaction. By applying this workflow, one could identify the suitability of materials for given photocatalytic reactions simply through a set of three experiments, to avoid unnecessary ad-hoc trials. 


\section{Conclusions}

In summary, we present a methodology employing a sequence of experimental techniques to determine the band structure of boron nitride and other metal-free photocatalysts on an absolute energy scale. We validated our methodology by comparing the experimentally obtained band structures for bulk graphitic carbon nitride $\left(\mathrm{g}-\mathrm{C}_{3} \mathrm{~N}_{4}\right)$ and amorphous boron with data from the literature. Following this methodology, we presented the complete band structure for different forms of boron nitride, a material that is gaining attention in the photocatalysis community, and aimed to provide additional insights into the photophysics of the material. Our approach can be extended to other porous metal-free photocatalysts and it offers a way to monitor systematic tuning and optimization of the photochemistry of such materials. This approach allows one to easily screen a range of porous metal-free photocatalysts for different reactions.

\section{Acknowledgements}

The authors would like to acknowledge the funding from EPSRC through the Doctoral Partnership fund (1855454), which made this research possible. We also acknowledge support from the Department of Chemical Engineering at Imperial College London.

\section{Declaration}

The authors declare that there are no competing financial interests.

\section{References}

1. G. Centi and S. Perathoner, ChemSusChem, 2010, 3, 195-208.

2. G. Centi, E. A. Quadrelli and S. Perathoner, Energy Environ. Sci., 2013, 6, 1711-1731.

3. S. Zhou, Y. Liu, J. Li, Y. Wang, G. Jiang, Z. Zhao, D. Wang, A. Duan, J. Liu and Y. Wei, Appl. Catal. B, 2014, 158, 20-29.

4. M. Zhou, S. Wang, P. Yang, C. Huang and X. Wang, ACS Catal., 2018, 8, 4928-4936. 
5. Z. Sun, J. M. T. A. Fischer, Q. Li, J. Hu, Q. Tang, H. Wang, Z. Wu, M. Hankel, D. J. Searles and L. Wang, Appl. Catal. B, 2017, 216, 146-155.

6. J. Lin, Z. Pan and X. Wang, ACS Sustain. Chem. Eng., 2013, 2, 353-358.

7. Y. Zhang and M. Antonietti, Chem. Asian J., 2010, 5, 1307-1311.

8. S. Yang, W. Hu, X. Zhang, P. He, B. Pattengale, C. Liu, M. Cendejas, I. Hermans, X. Zhang and J. Zhang, J. Am. Chem. Soc, 2018, 140, 14614-14618.

9. M. Lu, J. Liu, Q. Li, M. Zhang, M. Liu, J. L. Wang, D. Q. Yuan and Y. Q. Lan, Angew. Chem., 2019, 131, 12522-12527.

10. W. Zhong, R. Sa, L. Li, Y. He, L. Li, J. Bi, Z. Zhuang, Y. Yu and Z. Zou, J. Am. Chem. Soc, 2019, $141,7615-7621$.

11. R. S. Sprick, B. Bonillo, R. Clowes, P. Guiglion, N. J. Brownbill, B. J. Slater, F. Blanc, M. A. Zwijnenburg, D. J. Adams and A. I. Cooper, Angew. Chem. Int. Ed., 2016, 55, 1792-1796.

12. M. Sachs, R. S. Sprick, D. Pearce, S. A. Hillman, A. Monti, A. A. Guilbert, N. J. Brownbill, S. Dimitrov, X. Shi and F. Blanc, Nat. Commun, 2018, 9, 1-11.

13. R. S. Sprick, B. Bonillo, M. Sachs, R. Clowes, J. R. Durrant, D. J. Adams and A. I. Cooper, ChemComm, 2016, 52, 10008-10011.

14. L. Wang, R. Fernández-Terán, L. Zhang, D. L. Fernandes, L. Tian, H. Chen and H. Tian, Angew. Chem. Int. Ed., 2016, 55, 12306-12310.

15. A. Crake, K. C. Christoforidis, A. Gregg, B. Moss, A. Kafizas and C. Petit, Small, 2019, 15, 1805473.

16. A. Crake, K. C. Christoforidis, A. Kafizas, S. Zafeiratos and C. Petit, Appl. Catal. B, 2017, 210, 131-140.

17. R. Shankar, M. Sachs, L. Francàs, D. Lubert-Perquel, G. Kerherve, A. Regoutz and C. Petit, J. Mater. Chem. A, 2019, 7, 23931-23940.

18. M. Wang, M. Li, L. Xu, L. Wang, Z. Ju, G. Li and Y. Qian, Catal. Sci. Technol., 2011, 1, 11591165.

19. X. Li, J. Zhao and J. Yang, Sci. Rep., 2013, 3, 1858.

20. J. Pang, Y. Chao, H. Chang, H. Li, J. Xiong, M. He, Q. Zhang, H. Li and W. Zhu, J. Colloid Sci., 2017, 508, 121-128.

21. X. Wang, K. Maeda, A. Thomas, K. Takanabe, G. Xin, J. M. Carlsson, K. Domen and M. Antonietti, Nat. Mater, 2009, 8, 76.

22. Q. Weng, D. G. Kvashnin, X. Wang, O. Cretu, Y. Yang, M. Zhou, C. Zhang, D. M. Tang, P. B. Sorokin and Y. Bando, Adv. Mater., 2017, 29, 1700695.

23. W. J. Albery, G. J. O'Shea and A. L. Smith, J. Chem. Soc. Faraday Trans., 1996, 92, 4083-4085.

24. F. Fabregat-Santiago, G. Garcia-Belmonte, J. Bisquert, P. Bogdanoff and A. Zaban, J. Electrochem. Soc, 2003, 150, E293-E298.

25. C. Ong, S. Dennison, S. Fearn, K. Hellgardt and G. Kelsall, Electrochim. Acta, 2014, 125, 266274.

26. A. Hankin, F. Bedoya-Lora, C. Ong, J. Alexander, F. Petter and G. Kelsall, Energy Environ. Sci., 2017, 10, 346-360.

27. F. E. Bedoya-Lora, A. Hankin, I. Holmes-Gentle, A. Regoutz, M. Nania, D. J. Payne, J. T. Cabral and G. H. Kelsall, Electrochim. Acta, 2017, 251, 1-11.

28. E. M. Dias, K. C. Christoforidis, L. Francas and C. Petit, ACS Appl. Energy Mater., 2018, 1, 6524-6534.

29. R. Shankar, S. Marchesini and C. Petit, J. Phys. Chem. C, 2019, 123, 4282-4290.

30. M. Florent and T. J. Bandosz, J. Mater. Chem. A, 2018, 6, 3510-3521.

31. A. Hankin, F. E. Bedoya-Lora, J. C. Alexander, A. Regoutz and G. H. Kelsall, J. Mater. Chem. A, 2019, 7, 26162-26176.

32. A. Hankin, J. Alexander and G. Kelsall, Phys. Chem. Chem. Phys, 2014, 16, 16176-16186.

33. H. Zhang, X. Zuo, H. Tang, G. Li and Z. Zhou, Phys. Chem. Chem. Phys, 2015, 17, 6280-6288.

34. S. Hu, F. Li, Z. Fan, F. Wang, Y. Zhao and Z. Lv, Dalton Trans., 2015, 44, 1084-1092. 
35. L. Ma, H. Fan, M. Li, H. Tian, J. Fang and G. Dong, J. Mater. Chem. A, 2015, 3, 22404-22412.

36. G. A. Ozin, Adv. Mater., 2015, 27, 1957-1963.

37. O. Madelung, U. Rossler and M. Schulz, Non-Tetrahedrally Bonded Elements and Binary Compounds, Springer, 1998.

38. X.-F. Zhou, A. R. Oganov, Z. Wang, I. A. Popov, A. I. Boldyrev and H.-T. Wang, Phys. Rev. B, 2016, 93, 085406.

39. K. Shirai, J. Superhard Mater., 2010, 32, 205-225.

40. G. Liu, L. C. Yin, P. Niu, W. Jiao and H. M. Cheng, Angew. Chem. Int. Ed., 2013, 52, 6242-6245.

41. C. Maheu, L. Cardenas, E. Puzenat, P. Afanasiev and C. Geantet, Phys. Chem. Chem. Phys, 2018, 20, 25629-25637.

42. D. Cahen and A. Kahn, Adv. Mater., 2003, 15, 271-277.

43. H. Ishii, K. Sugiyama, E. Ito and K. Seki, Adv. Mater., 1999, 11, 605-625.

44. A. Kahn, Mater. Horiz, 2016, 3, 7-10.

45. J. Mizsei, Journal of Thin Solid Films, 2007, 515, 8310-8315.

46. A. Cowley and S. Sze, J. Appl. Phys., 1965, 36, 3212-3220.

47. S. Marchesini, C. M. McGilvery, J. Bailey and C. Petit, ACS Nano, 2017, 11, 10003-10011.

48. S. Brunauer, P. H. Emmett and E. Teller, J. Am. Chem. Soc, 1938, 60, 309-319.

49. S. Chen and R. Yang, Langmuir, 1994, 10, 4244-4249.

50. P. C. Rao and S. Mandal, CrystEngComm, 2014, 16, 9320-9325.

51. T. Sreekanth, P. Nagajyothi, G. Dillip and Y. R. Lee, J. Phys. Chem. C, 2017, 121, 25229-25242.

52. G. Liu, X. Meng, H. Zhang, G. Zhao, H. Pang, T. Wang, P. Li, T. Kako and J. Ye, Angew. Chem. Int. Ed., 2017, 56, 5570-5574.

53. N. Morita and A. Yamamoto, Jpn. J. Appl. Phys, 1975, 14, 825.

54. H. Lin, Y. Liu, J. Deng, S. Xie, X. Zhao, J. Yang, K. Zhang, Z. Han and H. Dai, J. Photochem. Photobiol, 2017, 336, 105-114.

55. A. Cros, J. Electron Spectros. Relat. Phenomena, 1992, 59, 1-14.

56. H. Z. Zeng, W. X. Zhang and W. L. Zhang, 2019.

57. J. Fang, H. Fan, M. Li and C. Long, J. Mater. Chem. A, 2015, 3, 13819-13826.

58. S. W. King, M. French, G. Xu, B. French, M. Jaehnig, J. Bielefeld, J. Brockman and M. Kuhn, Appl. Surf. Sci., 2013, 285, 545-551.

59. R. Schlaf, H. Murata and Z. Kafafi, J. Electron Spectros. Relat. Phenomena, 2001, 120, 149154.

60. J. Fuggle and D. Menzel, Surf. Sci., 1975, 53, 21-34.

61. L. Sygellou, G. Paterakis, C. Galiotis and D. Tasis, J. Phys. Chem. C, 2015, 120, 281-290.

62. M. Helander, M. Greiner, Z. Wang and Z. Lu, Appl. Surf. Sci., 2010, 256, 2602-2605.

63. S. Corby, E. Pastor, Y. Dong, X. Zheng, L. Francàs, M. Sachs, S. Selim, A. Kafizas, A. A. Bakulin and J. R. Durrant, Physical Chemistry Letters, 2019, 10, 5395-5401.

64. X. Ma, Y. Wei, Z. Wei, H. He, C. Huang and Y. Zhu, J. Colloid Sci., 2017, 508, 274-281.

65. X. Li, Y. Wang, W. Tian and J. Cao, ACS Omega, 2019, 4, 9645-9653.

66. R. Strayer, W. Mackie and L. Swanson, Surf. Sci., 1973, 34, 225-248.

67. S. Trasatti, Pure Appl. Chem, 1986, 58, 955-966.

68. A. U. Pawar, C. W. Kim, M.-T. Nguyen-Le and Y. S. Kang, ACS Sustain. Chem. Eng., 2019, 7, 7431-7455.

69. S. G. Bratsch, J. Phys. Chem. Ref. Data, 1989, 18, 1-21.

70. Y. Zheng, Z. Zhang and C. Li, J. Photochem. Photobiol, 2017, 332, 32-44.

71. T. Knežević, T. Suligoj and L. K. Nanver, presented in part at the 2019 42nd International Convention on Information and Communication Technology, Electronics and Microelectronics (MIPRO), 2019.

72. J. Qin, S. Wang, H. Ren, Y. Hou and X. Wang, Appl. Catal. B, 2015, 179, 1-8.

73. Y. Cui, J. Zhang, G. Zhang, J. Huang, P. Liu, M. Antonietti and X. Wang, J. Mater. Chem., 2011, 21, 13032-13039. 
74. X. Wang, S. Blechert and M. Antonietti, ACS Catal., 2012, 2, 1596-1606.

75. S. Duhm, G. Heimel, I. Salzmann, H. Glowatzki, R. L. Johnson, A. Vollmer, J. P. Rabe and N. Koch, Nat. Mater, 2008, 7, 326-332.

76. J. Topping, Proc. R. Soc. Lond., 1927, 114, 67-72. 